\title{
Ergodic Secrecy Capacity of RIS-Assisted Communication Systems in the Presence of Discrete Phase Shifts and Multiple Eavesdroppers
}

\author{
Peng Xu, Member, IEEE, Gaojie Chen, Senior Member, IEEE, Gaofeng Pan, Senior Member, IEEE, \\ and Marco Di Renzo, Fellow, IEEE
}

\begin{abstract}
This letter investigates the ergodic secrecy capacity (ESC) of a reconfigurable intelligent surface (RIS)-assisted communication system in the presence of discrete phase shifts and multiple eavesdroppers (Eves). In particular, a closed-form approximation of the ESC is derived for both non-colluding and colluding Eves. The analytical results are shown to be accurate when the number of reflecting elements of the RIS $N$ is large. Asymptotic analysis is provided to investigate the impact of $N$ on the ESC, and it is proved that the ESC scales with $\log _{2} \mathrm{~N}$ for both non-colluding and colluding Eves. Numerical results are provided to verify the analytical results and the obtained scaling laws.
\end{abstract}

Index Terms-Reconfigurable intelligent surface, discrete phase shifts, multiple eavesdroppers, ergodic secrecy capacity.

\section{INTRODUCTION}

Reconfigurable intelligent surfaces (RISs) utilize a large number of passive reflecting elements to customize wireless communication environments [1]-[4]. In particular, the amplitude and phase shift of each reflecting element can be controlled, so that the received signal at the intended receiver can be strengthened through passive beamforming [5]. Due to the low-cost, high energy-efficiency and full-duplex advantages, RISs are regarded as a promising technology for the next-generation wireless communications and hence have recently received much academic and industrial attention [6][8].

RISs have various potential applications in wireless communications, which include the design of secure wireless systems based on the concept of physical layer security (e.g., [9]-[14]). In [9]-[12], the authors investigated optimization problems to jointly design the beamforming vectors and phase shifts at the transmitter and RIS, respectively. In general, there exist two objectives for the design of the phase shifts at the RIS: (i) to strengthen the legitimate channels by co-phasing the reflected signals with the signal directly received from the transmitter; and (ii) to suppress the eavesdropping channels by setting the reflected signals at the eavesdroppers (Eves)

P. Xu is with Chongqing Key Laboratory of Mobile Communications Technology, School of Communication and Information Engineering, Chongqing University of Posts and Telecommunications, Chongqing, 400065, China. (email: xupeng@cqupt.edu.cn).

G. Chen is with School of Engineering, University of Leicester, Leicester LE1 7RH, U.K. (e-mail: gaojie.chen@leicester.ac.uk).

G. Pan is with the School of Information and Electronics Engineering, Beijing Institute of Technology, Beijing 100081, China, and he is also with Computer, Electrical and Mathematical Sciences and Engineering Division, King Abdullah University of Science and Technology (KAUST), Thuwal 23955-6900, Saudi Arabia. (e-mail: gaofeng.pan.cn@ieee.org).

M. D. Renzo is with Université Paris-Saclay, CNRS and CentraleSupélec, Laboratoire des Signaux et Systèmes, Gif-sur-Yvette, France. (email: marco.direnzo@centralesupelec.fr). to be in opposite phase with respect to the signal from the transmitter. The key idea behind the optimization problems in the existing works [9]-[12] lies in achieving a favorable trade-off between these two design objectives, which requires the knowledge of the instantaneous eavesdropping channel state information (CSI) at the transmitter and RIS. However, the instantaneous eavesdropping CSI is difficult to obtain in practice, since the Eves are usually passive and do not actively communicate with other nodes. Motivated by this consideration, the authors of [13] and [14] considered RIS-assisted secrecy communications without assuming the knowledge of the instantaneous eavesdropping CSI. In particular, the authors of [13] proposed a joint beamforming and jamming scheme to enhance the secrecy rate, and the authors of [14] analyzed the secrecy outage probability at the RIS. However, these two works only considered a single eavesdropper and assumed continuous phase shifts at the reflecting elements of the RIS.

Different from these existing works, this letter investigates the ergodic secrecy capacity (ESC) of RIS-assisted systems in the presence of discrete phase shifts and multiple Eves. In particular, by approximately characterizing the distribution of the received signal-to-noise-ratios (SNRs) at the Eves, we obtain a closed-form approximation of the ESC for both noncolluding and colluding Eves. In order to provide insights, asymptotic analysis is also provided from the obtained ESC, and it is proved that, as the number of reflecting elements $N$ goes to infinity, the ESC scales with $\log N$ for both noncolluding and colluding Eves. Numerical results are illustrated to verify that the analytical results and the scaling laws are accurate for large values of $N$.

Notation: $\mathbb{C}$ and $\mathbb{Z}$ denote the complex domain and integer set, respectively; for brevity, we denote $[1: M] \triangleq\{1, \ldots, M\}$, where $M$ is a positive integer, and $[x]^{+} \triangleq \max \{0, x\} ; \mathcal{C N}$ denotes the complex Gaussian distributions; $\mathbb{E}[\cdot]$ denotes the expectation of a random variable; $\log (\cdot)$ and $\ln (\cdot)$ denote the base-two and natural logarithms, respectively; and $\kappa$ is Euler's constant.

\section{System Model AND PRELIMINARIES}

As shown in Fig. 1, we consider an RIS-assisted secure communication system with a source $(S)$, an RIS $(R)$ with $N$ reflecting elements, a destination $(D)$ and $K$ Eves $\left(E_{k}\right.$, $\forall k \in[1: K])$. All nodes are assumed to be equipped with a single antenna. The channels $S \rightarrow D, S \rightarrow E_{k}, S \rightarrow R$, $R \rightarrow D$ and $R \rightarrow E_{k}$ are denoted by $h_{S D} \in \mathbb{C}, h_{S E_{k}} \in \mathbb{C}$, $\mathbf{h}_{S R} \in \mathbb{C}^{N \times 1}, \mathbf{h}_{R D} \in \mathbb{C}^{N \times 1}$ and $\mathbf{h}_{k} \in \mathbb{C}^{N \times 1}$, respectively. These channels are modeled as $h_{S D}=g_{S D} d_{S D}^{-\frac{\alpha}{2}}, h_{S E_{k}}=$ $g_{S E_{k}} d_{S E_{k}}^{-\frac{\alpha}{2}},\left[\mathbf{h}_{S R}\right]_{n}=g_{S R, n} d_{S R}^{-\frac{\alpha}{2}},\left[\mathbf{h}_{R D}\right]_{n}=g_{R D, n} d_{R D}^{-\frac{\alpha}{2}}$ and 


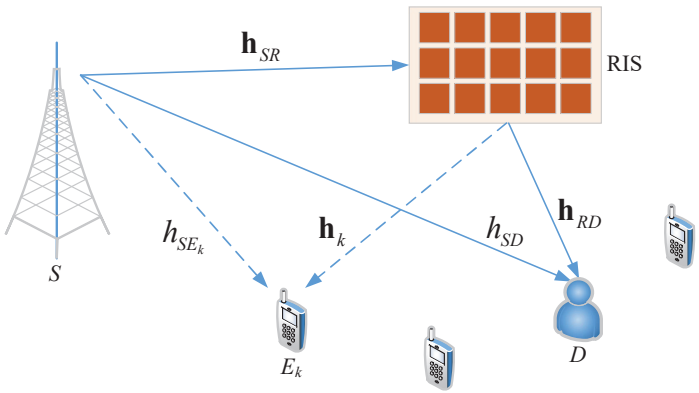

Fig. 1. RIS-assisted secrecy communication system.

$\left[\mathbf{h}_{k}\right]_{n}=g_{k, n} d_{k}^{-\frac{\alpha}{2}}$, where $g_{S D}, g_{S E_{k}}, g_{S R, n}, g_{R D, n}, g_{k, n} \sim$ $\mathcal{C N}(0,1)$ denote the small-scale fading, $d_{S D}, d_{S E_{k}}, d_{S R}, d_{R D}$ and $d_{k}$ denote the distances $S \rightarrow D, S \rightarrow E_{k}, S \rightarrow R$, $R \rightarrow D$ and $R \rightarrow E_{k}$, respectively, $\forall n \in[1: N], k \in[1: K]$, and $\alpha$ is the pass-loss exponent. Then, the received signal at $D$ and $E_{k}$ can be written as

$$
\begin{aligned}
y_{D} & =\sqrt{P}\left(\eta \mathbf{h}_{S R}^{T} \boldsymbol{\Phi} \mathbf{h}_{R D}+h_{S D}\right) x_{S}+n_{D}, \\
y_{E_{k}} & =\sqrt{P}\left(\eta \mathbf{h}_{S R}^{T} \boldsymbol{\Phi} \mathbf{h}_{k}+h_{S E_{k}}\right) x_{S}+n_{E_{k}},
\end{aligned}
$$

respectively, where $x_{S}$ is the transmitted signal, $\mathbb{E}\left(\left|x_{S}\right|^{2}\right)=1$, $P$ is the transmit power, $n_{D}$ and $n_{E_{k}} \sim \mathcal{C N}\left(0, \delta^{2}\right)$ are the additive white Gaussian noises at $D$ and $E_{k}$, respectively, $\eta \in(0,1]$ is the amplitude reflection coefficient, $\Phi \triangleq \operatorname{diag}\left(e^{j \phi_{1}}, \ldots, e^{j \phi_{N}}\right)$ and $\phi_{n} \in[0,2 \pi)$ is the $n$th phase shift at the RIS. We assume that the RIS does not have access to the instantaneous eavesdropping CSI, so that it cannot design $\phi_{n}$ in order to suppress the received SNRs at the Eves. However, the RIS is assumed to know the instantaneous legitimate CSI. Under these assumptions, the optimal value of $\phi_{n}$ that maximizes the received SNR at $D$ is $\phi_{n}^{*}=\theta_{S D}-\theta_{S R, n}-\theta_{R D, n}$, where $\theta_{S D}, \theta_{S R, n}$ and $\theta_{R D, n}$ denote the phases of $g_{S D}, g_{S R, n}$ and $g_{R D, n}$, respectively. Furthermore, in this letter, $\phi_{n}$ can only take a finite number of discrete values. In particular, the set of discrete phase shifts is denoted by $\mathcal{F} \triangleq\left\{0, \frac{2 \pi}{2^{b}}, \ldots, \frac{\left(2^{b}-1\right) 2 \pi}{2^{b}}\right\}$, where $b$ denotes the number of quantization bits. Accordingly, we set $\phi_{n}=f_{1}\left(\phi_{n}^{*}\right)$, where the function $f_{1}\left(\phi_{n}^{*}\right)$ maps $\phi_{n}^{*}$ to the nearest point in $\mathcal{F}$, i.e.,

$$
f_{1}\left(\phi_{n}^{*}\right)=\hat{\phi}_{i}, \text { if }\left|\phi_{n}^{*}-\hat{\phi}_{i}\right| \leq\left|\phi_{n}^{*}-\hat{\phi}_{j}\right|, \hat{\phi}_{i}, \hat{\phi}_{j} \in \mathcal{F}, \forall j \neq i \text {. }
$$

Therefore, the quantization error is $\Theta_{n}=f_{1}\left(\phi_{n}^{*}\right)-\phi_{n}^{*}$, which is uniformly distributed in $\left[-\frac{\pi}{2^{b}}, \frac{\pi}{2^{b}}\right]$, similar to [15][18]. Accordingly, the received SNRs at $D$ and $E_{k}$ can be formulated, respectively, as follows

$$
\begin{aligned}
\gamma_{D} & =\rho|| h_{S D}\left|+\eta \sum_{n=1}^{N}\right|\left[\mathbf{h}_{S R}\right]_{n}\left[\mathbf{h}_{R D}\right]_{n}\left|e^{j \Theta_{n}}\right|^{2} \\
& =\rho\left|d_{S D}^{-\frac{\alpha}{2}}\right| g_{S D}\left|+\eta d_{S R}^{-\frac{\alpha}{2}} d_{R D}^{-\frac{\alpha}{2}} \sum_{n=1}^{N}\right| g_{S R, n} g_{R D, n}\left|e^{j \Theta_{n}}\right|^{2}, \\
\gamma_{E_{k}} & =\rho\left|h_{S E_{k}}+\eta \sum_{n=1}^{N}\right|\left[\mathbf{h}_{S R}\right]_{n}\left[\mathbf{h}_{k}\right]_{n}\left|e^{j \psi_{k, n}}\right|^{2} \\
& =\rho\left|d_{S E_{k}}^{-\frac{\alpha}{2}} g_{S E_{k}}+\eta d_{S R}^{-\frac{\alpha}{2}} d_{k}^{-\frac{\alpha}{2}} \sum_{n=1}^{N}\right| g_{S R, n} g_{k, n}\left|e^{j \psi_{k, n}}\right|^{2},
\end{aligned}
$$

where $\psi_{k, n} \triangleq f_{2}\left(\phi_{n}^{*}, \theta_{S R, n}\right)+\theta_{k, n}, \theta_{k, n}$ is the phase of $g_{k, n}$ and the function $f_{2}\left(\phi_{n}^{*}, \theta_{S R, n}\right)$ is defined as follows

$$
f_{2}\left(\phi_{n}^{*}, \theta_{S R, n}\right) \triangleq f_{1}\left(\phi_{n}^{*}\right)+\theta_{S R, n} .
$$

The ESC can be expressed as follows [19]

$$
C_{s}=\left[C_{D}-C_{E}\right]^{+},
$$

where $C_{D}=\mathbb{E}_{\gamma_{D}}\left[\log \left(1+\gamma_{D}\right)\right]$ and $C_{E}$ denote the ergodic capacities from $S$ to $D$ and the Eves, respectively. Given $\left\{\Theta_{n}\right\}_{n=1}^{N}$, an approximated expression of $C_{D}$ can be found in [16, Eq. (13)]. By averaging over $\{\Theta\}_{n=1}^{N}, C_{D}$ can be calculated as shown in (8) at the top of the next page.

In the following sections, $C_{E}$ is calculated for non-colluding and colluding Eves, respectively.

\section{Non-COLluding Eves}

In the non-colluding case, $C_{E}$ can be expressed as follows

$$
C_{E}=\max _{k \in[1: K]} C_{E_{k}}
$$

where $C_{E_{k}} \triangleq \mathbb{E}_{\gamma_{E_{k}}}\left[\log \left(1+\gamma_{E_{k}}\right)\right]$. In order to derive $C_{E_{k}}$, the distribution of $\gamma_{E_{k}}$ in (5) needs to be computed.

\section{A. Distribution of $\gamma_{E_{k}}$}

Before deriving the distribution of $\gamma_{E_{k}}$, we introduce the following lemma.

Lemma 1: The phase $\psi_{k, n}, k \in[1: K], n \in[1: N]$, in (5) has the following properties:

a) $\psi_{k, n}$ is uniformly distributed in $[0,2 \pi)$;

b) $\psi_{k, n}$ is independent of $f_{2}\left(\phi_{n}^{*}, \theta_{S R, n}\right)$ defined in (6);

c) $\psi_{k, i}$ is independent of $\psi_{k, j}, \forall i \neq j, i, j \in[1: N]$.

Proof 1: See Appendix A

Based on Lemma1, the distribution of $\gamma_{E_{k}}$ in (5) is provided in the following lemma.

Lemma 2: When $N$ is large, $\gamma_{E_{k}}$ can be approximated as an exponential random variable with mean $\lambda_{E_{k}}=\rho\left(d_{S E_{k}}^{-\alpha}+\right.$ $N B_{k}$ ), where $B_{k} \triangleq \eta^{2} d_{S R}^{-\alpha} d_{k}^{-\alpha}$.

Proof 2: Define $G_{k} \triangleq \sum_{n=1}^{N}\left|g_{S R, n} g_{k, n}\right| e^{j \psi_{k, n}}, k \in[1:$ $K]$. Based on [20, Lemma 2] and the fact that $\left\{\psi_{k, n}\right\}_{n=1}^{N}$ are independent and identically distributed (i.i.d.) uniform random variables in $[0,2 \pi)$ as proved in Lemma 1, $G_{k} \sim \mathcal{C N}(0, N)$ as $N \rightarrow \infty$. Furthermore, since $g_{S E_{k}}$ is independent of $G_{k}$, we have

$$
d_{S E_{k}}^{-\frac{\alpha}{2}} g_{S E_{k}}+\eta d_{S R}^{-\frac{\alpha}{2}} d_{k}^{-\frac{\alpha}{2}} G_{k} \sim \mathcal{C N}\left(0, d_{S E_{k}}^{-\alpha}+N B_{k}\right),
$$

as $N \rightarrow \infty$. Recalling that $\gamma_{E_{k}}=\rho\left|d_{S E_{k}}^{-\frac{\alpha}{2}} g_{S E_{k}}+\sqrt{B_{k}} G_{k}\right|^{2}$ in (5), the proof follows.

\section{B. Ergodic Secrecy Capacity}

The ESC for non-colluding Eves is summarized in the following theorem.

Theorem 1: When $N$ is large, the ESC for non-colluding Eves can be expressed as follows

$$
C_{s} \approx\left[C_{D}+\frac{1}{\ln 2} \max _{k \in[1: K]} e^{\frac{1}{\lambda_{E_{k}}}} \operatorname{Ei}\left(-\frac{1}{\lambda_{E_{k}}}\right)\right]^{+},
$$

where $\operatorname{Ei}(\mathrm{x}) \triangleq-\int_{-\mathrm{x}}^{\infty} \frac{\mathrm{e}^{-\mathrm{t}}}{\mathrm{t}} \mathrm{dt}, x<0$, is the exponential integral function [21, Eq. 8.211]. 


$$
\begin{aligned}
C_{D} & \approx \log \left(1+\rho\left(N \eta^{2} d_{S R}^{-\alpha} d_{R D}^{-\alpha}+d_{S D}^{-\alpha}+\frac{\pi^{\frac{3}{2}} \eta}{4} d_{S D}^{-\frac{\alpha}{2}} d_{S R}^{-\frac{\alpha}{2}} d_{R D}^{-\frac{\alpha}{2}} \sum_{n=1}^{N} \mathbb{E}_{\Theta_{n}}\left[\cos \Theta_{n}\right]+\frac{\pi^{2} \eta^{2}}{8} d_{S R}^{-\alpha} d_{R D}^{-\alpha} \sum_{i=1}^{N-1} \sum_{k=i+1}^{N} \mathbb{E}_{\Theta_{i}, \Theta_{k}}\left[\cos \left(\Theta_{k}-\Theta_{i}\right)\right]\right)\right) \\
& =\log \left(1+\rho N A_{1}+\rho d_{S D}^{-\alpha}+\rho N A_{2}+\rho N(N-1) A_{3}\right),
\end{aligned}
$$

where $A_{1} \triangleq \eta^{2} d_{S R}^{-\alpha} d_{R D}^{-\alpha}, A_{2} \triangleq \frac{\sqrt{\pi} \eta 2^{b}}{4} d_{S D}^{-\frac{\alpha}{2}} d_{S R}^{-\frac{\alpha}{2}} d_{R D}^{-\frac{\alpha}{2}} \sin \frac{\pi}{2^{b}}$ and $A_{3} \triangleq \frac{\eta^{2} 2^{2 b}}{32} d_{S R}^{-\alpha} d_{R D}^{-\alpha}\left(1-\cos \frac{2 \pi}{2^{b}}\right)$.

Proof 3: When $N$ is large, based on Lemma 2 . $C_{E_{k}}$ in (9) can be approximated as follows

$$
\begin{aligned}
C_{E_{k}} & \approx \int_{0}^{\infty} \log (1+x) \frac{1}{\lambda_{E_{k}}} e^{-\frac{x}{\lambda_{E_{k}}}} \mathrm{~d} x=\frac{1}{\ln 2} \int_{0}^{\infty} \frac{e^{-\frac{x}{\lambda_{E_{k}}}}}{1+x} \mathrm{~d} x \\
& =-\frac{e^{\frac{1}{\lambda_{E_{k}}}}}{\ln 2} \operatorname{Ei}\left(-\frac{1}{\lambda_{E_{k}}}\right),
\end{aligned}
$$

where the last equality is based on [21, Eq. 3.352.4]. Combining (7), (9) and (11), the theorem is proved.

\section{Asymptotic Analysis}

To obtain insights from the obtained ESC, its asymptotic behavior is analyzed in the following corollary.

Corollary 1: As $N \rightarrow \infty, C_{s} \rightarrow \log N+\log A_{3}+$ $\frac{\kappa}{\ln 2}-\max _{k \in[1: K]} \log B_{k}$, which implies that the ESC for noncolluding Eves scales with $\log N$.

Proof 4: From [8], we have

$$
\begin{aligned}
C_{D} & \approx \log \left(\rho A_{3} N^{2}\left(\frac{1}{\rho A_{3} N^{2}}+\frac{A_{1}}{A_{3} N}+\frac{d_{S D}^{-\alpha}}{A_{3} N^{2}}+\frac{A_{2}-A_{3}}{A_{3} N}+1\right)\right) \\
& \rightarrow 2 \log N+\log \rho+\log A_{3}, \text { as } N \rightarrow \infty .
\end{aligned}
$$

In addition, $C_{E_{k}}$ in (11) can be further expressed as follows

$$
\begin{aligned}
C_{E_{k}} & \stackrel{(a)}{\approx} \frac{e^{\frac{1}{\lambda_{E_{k}}}}}{\ln 2}\left(-\kappa+\ln \left(\lambda_{E_{k}}\right)+\sum_{i=1}^{\infty} \frac{(-1)^{i+1}}{i \cdot i ! \cdot \lambda_{E_{k}}^{i}}\right) \\
& \stackrel{(b)}{\rightarrow} \log \left(\lambda_{E_{k}}\right)-\frac{\kappa}{\ln 2} \\
& \stackrel{(c)}{=} \log \left(N \rho B_{k}\right)+\log \left(1+\frac{d_{S E_{k}}^{-\alpha}}{N B_{k}}\right)-\frac{\kappa}{\ln 2} \\
& \rightarrow \log N+\log \rho+\log B_{k}-\frac{\kappa}{\ln 2}, \text { as } N \rightarrow \infty .
\end{aligned}
$$

where $(a)$ is based on (11) and [21, Eq. 8.214.1], (b) holds since $1 / \lambda_{E_{k}} \rightarrow 0$ as $N \rightarrow \infty$, and (c) is based on the definition of $\lambda_{E_{k}}$ in Lemma 2

Combining (7), (9), (12) and (13), this corollary follows.

Remark 1: Compared with the scaling $\operatorname{law} 2 \log N$ for non-secrecy transmission with discrete phase shifts [15], [16], Corollary 1 shows that the ESC has a different scaling law that is equal to $\log N$.

\section{Colluding Eves}

When the Eves are colluding, they can combine their received signals for information interception. Thus, the $C_{E}$ in (7) can be expressed as follows

$$
C_{E}=\mathbb{E}_{\left\{\gamma_{E_{k}}\right\}_{k=1}^{K}} \log \left(1+\sum_{k=1}^{K} \gamma_{E_{k}}\right) .
$$

Since common random variables $\left\{g_{S R, n}\right\}_{n=1}^{N}$ are present in every $\gamma_{E_{k}}$ as shown in (5), $\left\{\gamma_{E_{k}}\right\}_{k=1}^{K}$ are correlated random variables. However, the following lemma shows that such correlation is negligible for large values of $N$.

Lemma 3: $\gamma_{E_{i}}$ is independent of $\gamma_{E_{j}}$ if $N \rightarrow \infty, i, j \in$ $[1: K], i \neq j$.

Proof 5: See Appendix B

\section{A. Ergodic Secrecy Capacity}

Based on Lemma 2 and Lemma 3, the ESC is provided in the following theorem.

Theorem 2: When $N$ is large, and $\lambda_{E_{i}} \neq \lambda_{E_{j}}, \forall i \neq j$, $i, j \in[1: N]$, the ESC for colluding Eves can be approximated as follows

$$
C_{s} \approx\left[C_{D}+\frac{1}{\ln 2} \sum_{i=1}^{K} e^{\frac{1}{\lambda_{E_{i}}}} \operatorname{Ei}\left(-\frac{1}{\lambda_{E_{i}}}\right) \prod_{j=1, j \neq i}^{K} \frac{\lambda_{E_{i}}}{\lambda_{E_{i}}-\lambda_{E_{j}}}\right]^{+} .
$$

Proof 6: Based on Lemma 3, if $\lambda_{E_{i}} \neq \lambda_{E_{j}}, \forall i \neq j$, $\sum_{k=1}^{K} \gamma_{E_{k}}$ has the following probability density function (PDF) [22]:

$$
f_{\sum_{k=1}^{K} \gamma_{E_{k}}}(x)=\sum_{i=1}^{K} \frac{1}{\lambda_{E_{i}}} e^{-\frac{x}{\lambda_{E_{i}}}} \prod_{j=1, j \neq i}^{K} \frac{\lambda_{E_{i}}}{\lambda_{E_{i}}-\lambda_{E_{j}}} .
$$

Combining (11), 140 and (16), we obtain

$$
C_{E} \approx-\frac{1}{\ln 2} \sum_{i=1}^{K} e^{\frac{1}{\lambda_{E_{i}}}} \operatorname{Ei}\left(-\frac{1}{\lambda_{E_{i}}}\right) \prod_{j=1, j \neq i}^{K} \frac{\lambda_{E_{i}}}{\lambda_{E_{i}}-\lambda_{E_{j}}} .
$$

Recalling (7), we prove the theorem.

Remark 2: Theorem 2 corresponds to the case that the Eves lie in different locations, so that $\left\{\gamma_{E_{k}}\right\}_{k=1}^{K}$ have different means. When the Eves are clustered relatively closely together that $\left\{\gamma_{E_{k}}\right\}_{k=1}^{K}$ have the same mean, the ESC can be analyzed in a similar way, whose details are not provided due to space limitations.

\section{B. Asymptotic Analysis}

The asymptotic behavior of the obtained ESC for colluding Eves is provided in the following corollary.

Corollary 2: As $N \rightarrow \infty, C_{s} \rightarrow \log N+\log A_{3}+\frac{\kappa}{\ln 2}-$ $\sum_{i=1}^{K} \log B_{i} \prod_{j=1, j \neq i}^{K} \frac{B_{i}}{B_{i}-B_{j}}$, which implies that the ESC for colluding Eves also scales with $\log N$.

Proof 7: From (11), 113) and (17), we have

$$
\begin{aligned}
C_{E} & \rightarrow \sum_{i=1}^{K}\left(\log N+\log \rho-\frac{\kappa}{\ln 2}+\log B_{i}\right) \prod_{j=1, j \neq i}^{K} \frac{\lambda_{E_{i}}}{\lambda_{E_{i}}-\lambda_{E_{j}}} \\
& \stackrel{(a)}{\rightarrow} \sum_{i=1}^{K}\left(\log N+\log \rho-\frac{\kappa}{\ln 2}+\log B_{i}\right) \prod_{j=1, j \neq i}^{K} \frac{B_{i}}{B_{i}-B_{j}} \\
& \stackrel{(b)}{=} \log N+\log \rho-\frac{\kappa}{\ln 2}+\sum_{i=1}^{K} \log B_{i} \prod_{j=1, j \neq i}^{K} \frac{B_{i}}{B_{i}-B_{j}}, \quad(18)
\end{aligned}
$$




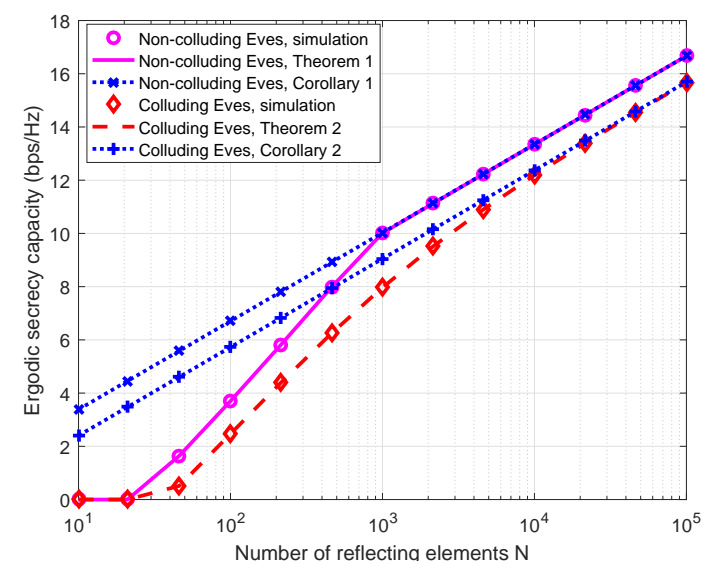

Fig. 2. Ergodic secrecy capacity vs. $N$, for $K=5$.

where $(a)$ holds since $\lambda_{E_{k}}=\rho N B_{k}\left(1+\frac{d_{S E_{k}}^{-\alpha}}{N B_{k}}\right) \rightarrow \rho N B_{k}$ as $N \rightarrow \infty$, and $(b)$ is based on the fact that $\sum_{i=1}^{K} \prod_{j=1, j \neq i}^{K} \frac{B_{i}}{B_{i}-B_{i}}=1$, as proved in [22, Chapter 5].

Combining (7), (12), (14) and (18), the proof follows.

Remark 3: Comparing Corollaries 1 and 2 we evince that only the last terms for the asymptotic ESC are different, i.e., $\max _{k \in[1: K]} \log B_{k}$ and $\sum_{i=1}^{K} \log B_{i} \prod_{j=1, j \neq i}^{K} \frac{B_{i}}{B_{i}-B_{j}}$ for noncolluding and colluding Eves, respectively. In addition, the ESCs for both non-colluding and colluding Eves have the same scaling $\operatorname{law} \log N$.

\section{Large Number of Eves}

To obtain more insights from the obtained ESC, we provide a simplified expression of $C_{s}$ for large values of $K$ in the following corollary.

Corollary 3: When $N$ and $K \rightarrow \infty$, the ESC for colluding Eves can be approximated as follows

$$
C_{s} \approx\left[C_{D}-\log \left(1+\sum_{k=1}^{K} \lambda_{E_{k}}\right)\right]^{+} .
$$

Proof
$\operatorname{Var}\left[\sum_{k=1}^{K} \gamma_{E_{k}}\right] \rightarrow \sum_{k=1}^{K} \lambda_{E_{k}}^{2}$ as $N \rightarrow \infty$ Lemma $\frac{\operatorname{Var}\left[\sum_{k=1}^{K} \gamma_{k}\right]}{\left(\mathbb{E}\left[\sum_{k=1}^{K} \gamma_{k}\right]\right)^{2}} \rightarrow \frac{\sum_{k=1}^{K} \lambda_{E_{k}}^{2}}{\left(\sum_{k=1}^{K} \lambda_{E_{k}}\right)^{2}} \rightarrow 0$ as $N$ and $K \rightarrow \infty$. According to [23, Theorem 4], $C_{E}$ in (14) can be approximated as follows

$$
C_{E} \approx \log \left(1+\mathbb{E}\left[\sum_{k=1}^{K} \gamma_{k}\right]\right) .
$$

This completes the proof.

\section{Numerical Results}

In this section, numerical results are provided to verify the analytical results in the obtained theorems and corollaries. The simulation results are averaged over $10^{4}$ channel realizations. For illustrative purposes, we set $\alpha=3, b=3$ bits, $P=20$ $\mathrm{dBm}, \sigma^{2}=-96 \mathrm{dBm}$ and $\eta=0.8$. In addition, $S, R$ and $D$ are located at $(0,0) \mathrm{m},(100,0) \mathrm{m}$ and $(90,20) \mathrm{m}$, respectively. As for the Eves, $E_{k}$ is located at $\left(\frac{90 k}{K},-20\right) \mathrm{m}, k \in[1: K]$. For the considered simulation configuration, the ESC is zero

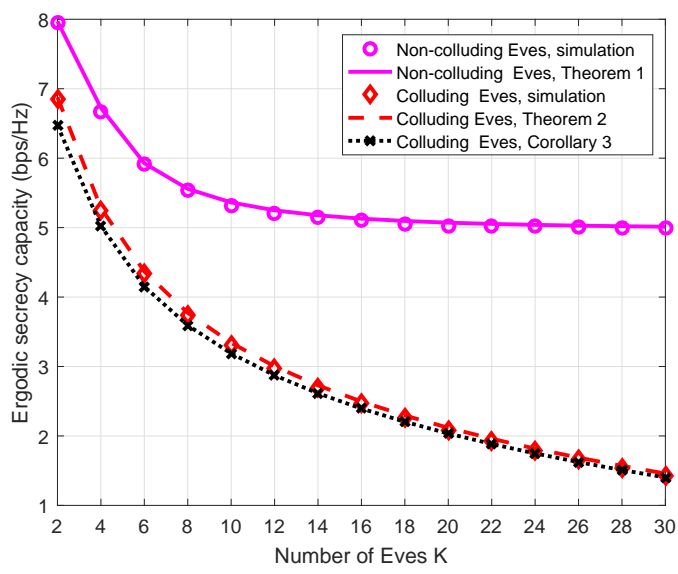

Fig. 3. Ergodic secrecy capacity vs. $K$, for $N=250$.

in the absence of the RIS. In this case, in fact, the ESC for non-colluding Eves can be expressed as follows

$$
\begin{aligned}
C_{s} & =\left[\mathbb{E}_{h_{S D}}\left[1+\rho\left|h_{S D}\right|^{2}\right]-\max _{k \in[1: K]} \mathbb{E}_{h_{S E_{k}}}\left[1+\rho\left|h_{S E_{k}}\right|^{2}\right]\right]^{+} \\
& =\left[f_{3}\left(d_{S D}\right)-\max _{k \in[1: K]} f_{3}\left(d_{S E_{k}}\right)\right]^{+},
\end{aligned}
$$

where $f_{3}(x) \triangleq \frac{1}{\ln 2} \int_{0}^{\infty} e^{-\frac{t}{\rho x^{-\alpha}}} /(1+t) \mathrm{d} t, x>0$. Therefore, $C_{s}=0$ in 21), since $f_{3}(x)$ is a decreasing function of $x$ and $d_{S D} \geq d_{S E_{k}}$ in the considered simulation configuration, $\forall k \in[1: K]$.

Fig. 2 2 shows the impact of the number of reflecting elements $N$ on the ESC, when the number of Eves is $K=5$. We can observe that the ESC increases with $N$. For example, the ESCs are about $3.7 \mathrm{bps} / \mathrm{Hz}$ and $2.5 \mathrm{bps} / \mathrm{Hz}$ for non-colluding and colluding Eves, respectively, if $N=100$. We observe that the approximated analytical results in Theorems 1 and 2 match well with Monte Carlo simulations. In addition, the asymptotic analytical results obtained in Corollaries 1 and 2 asymptotically approach the simulations as $N$ becomes sufficiently large, which confirms the scaling laws in Corollaries 1 and 2 The setup with non-colluding Eves provides a larger secrecy capacity since only the "best" Eve determines the ESC. There exists a constant gap of about $1 \mathrm{bps} / \mathrm{Hz}$ between the ESCs for non-colluding and colluding Eves if $N$ exceeds $10^{4}$.

In Fig. 3, the ESC is shown as a function of the number of Eves $K$, by assuming $N=250$. This figure confirms the findings in Corollary 3 for colluding Eves, and we observe that the approximation in (19) becomes tighter as $K$ increases. The ESCs for both non-colluding and colluding Eves decrease with $K$. However, the ESC for non-colluding Eves is less affected by $K$. We observe, in particular, that there exists an ESC floor of about $5 \mathrm{bps} / \mathrm{Hz}$ for large values of $K$. This is because the ESC for non-colluding Eves is determined by the nearest Eve to the source. In the considered simulation setup, the nearest Eve is located at around $(0,-20) \mathrm{m}$, when $K$ is large.

\section{Conclusions}

This letter investigated the ESC of an RIS-assisted communication system in the presence of discrete phase shifts and multiple Eves. We obtained an approximated closed-form 
expression of the ESC for both non-colluding and colluding Eves, which were demonstrated to be accurate for large values of the number of RIS reflecting elements. By analyzing the asymptotic behaviors of the obtained ESC, we unveiled that the ESC scales with $\log N$ in the presence of both non-colluding and colluding Eves. Numerical results were provided to verify the accuracy of the analytical results and the scaling laws.

\section{APPENDIX A \\ PROOF OF LEMMA 1}

Let us denote $X_{1, n} \triangleq f_{2}\left(\phi_{n}^{*}, \theta_{S R, n}\right), X_{2, n} \triangleq \theta_{k, n}$ and $Y_{n} \triangleq \psi_{k, n}$. Accordingly, $Y_{n}=X_{1, n}+X_{2, n}$ as shown in Section [II. We note that the random phases $X_{1, n}, X_{2, n}$ and $Y_{n}$ have a uniform circular distribution [15].

Given $X_{1, n}=x_{1}, \forall x_{1} \in[0,2 \pi), Y_{n}=x_{1}+X_{2, n}$ is uniformly distributed in $\left[x_{1}, x_{1}+2 \pi\right)=\left[x_{1}, 2 \pi\right) \cup\left[2 \pi, x_{1}+2 \pi\right)$. Since $Y_{n}$ has a circular uniform distribution, $\left[2 \pi, x_{1}+2 \pi\right)$ is equivalent to $\left[0, x_{1}\right)$. Thus, $Y_{n}$ is uniformly distributed in $[0,2 \pi)$, which proves Lemma 1-a).

Since $X_{1, n}$ and $X_{2, n}$ are independent, their joint PDF is

$$
f_{X_{1, n}, X_{2, n}}\left(x_{1}, x_{2}\right)=f_{X_{1, n}}\left(x_{1}\right) f_{X_{2, n}}\left(x_{2}\right)=\frac{1}{2 \pi} f_{X_{1, n}}\left(x_{1}\right) \text {. }
$$

We can construct the following Jacobian matrix

$$
J_{X_{1, n}, Y_{n}}\left(x_{1}, x_{2}\right)=\left[\begin{array}{ll}
\frac{\partial x_{1}}{\partial x_{1}}, & \frac{\partial x_{1}}{\partial x_{2}} \\
\frac{\partial y}{\partial x_{1}}, & \frac{\partial y}{\partial x_{2}}
\end{array}\right]=\left[\begin{array}{ll}
1, & 0 \\
1, & 1
\end{array}\right] .
$$

Thus, the joint PDF of $X_{1, n}$ and $Y_{n}$ can be written as

$$
\begin{aligned}
f_{X_{1, n}, Y_{n}}\left(x_{1}, y\right) & =\frac{f_{X_{1, n}, X_{2, n}}\left(x_{1}, x_{2}\right)}{\operatorname{det}\left(J_{X_{1, n}, Y_{n}}\left(x_{1}, x_{2}\right)\right)}=\frac{1}{2 \pi} f_{X_{1, n}}\left(x_{1}\right) \\
& =f_{X_{1, n}}\left(x_{1}\right) f_{Y_{n}}(y),
\end{aligned}
$$

which implies that $Y_{n}$ is independent of $X_{1, n}$. Thus, Lemma 1.b) is proved.

For $\forall i \neq j$ and $i, j \in[1: N]$, we have $Y_{i}=X_{1, i}+X_{2, i}$ and $Y_{j}=X_{1, j}+X_{2, j}$. Although the same random phase $\theta_{S D}$ is present in both $Y_{i}$ and $Y_{j}$ as shown in Section III $Y_{i}$ is still independent of $Y_{j}$, due to the following two facts: (i) $Y_{i}$ and $Y_{j}$ are independent of $X_{1, i}$ and $X_{1, j}$, respectively, according to Lemma 1.b); (ii) $X_{2, i}$ is independent of $X_{2, j}$. Therefore, Lemma 1-c) is proved.

\section{APPENDIX B}

\section{PROOF OF LEMMA 3}

$\begin{array}{clll}\text { Let us define } H_{E_{k}} \triangleq & d_{S E_{k}}^{-\frac{\alpha}{2}} g_{S E_{k}}+ \\ \sqrt{B_{k}} \sum_{n=1}^{N}\left|g_{S R, n} g_{k, n}\right| e^{j \psi_{k, n}} \text {. Thus, } \gamma_{E_{k}}=\rho\left|H_{E_{k}}\right|^{2} \text {. }\end{array}$ According to Lemma 1 -a), $\mathbb{E}\left[e^{j \psi_{k, n}}\right]=0$ and

$$
\mathbb{E}\left[H_{E_{k}}\right]=0, \forall k \in[1: K] .
$$

Moreover, since $\psi_{i, n}$ is independent of $\psi_{j, m}$ if $i \neq j$ or $n \neq$ $m$, we have

$$
\mathbb{E}\left[H_{E_{i}} H_{E_{j}}\right]=0, \forall i \neq j .
$$

From (25) and 26), the covariance of $H_{E_{i}}$ and $H_{E_{j}}$ can be expressed as follows

$$
\operatorname{Cov}\left(H_{E_{i}} H_{E_{j}}\right)=\mathbb{E}\left[H_{E_{i}} H_{E_{j}}\right]-\mathbb{E}\left[H_{E_{i}}\right] \mathbb{E}\left[H_{E_{j}}\right]=0 .
$$

${ }^{1}$ A random phase $X$ that follows a circular distribution fulfills the property that all the realization points $\{x+2 M \pi\}_{\forall M \in \mathbb{Z}}$ of $X$ are equivalent to the point $x$, where $x \in[0,2 \pi)$.
Based on Lemma 2 and (27), $\left\{H_{k}\right\}_{k=1}^{K}$ are uncorrelated complex Gaussian variables if $N \rightarrow \infty$, and hence $\left\{H_{k}\right\}_{k=1}^{K}$ are independent of each other. This completes the proof.

\section{REFERENCES}

[1] M. Di Renzo, et al., "Smart radio environments empowered by reconfigurable AI meta-surfaces: An idea whose time has come," EURASIP Journal on Wireless Communications and Networking, vol. 2019, no. 1, pp. 1-20, May 2019.

[2] — "Smart radio environments empowered by reconfigurable intelligent surfaces: How it works, state of research, and road ahead," arXiv:2004.09352.

[3] E. Basar, M. Di Renzo, J. De Rosny, M. Debbah, M. Alouini, and R. Zhang, "Wireless communications through reconfigurable intelligent surfaces," IEEE Access, vol. 7, pp. 116753-116773, Aug. 2019.

[4] M. Di Renzo, et al., "Reconfigurable intelligent surfaces vs. relaying: Differences, similarities, and performance comparison," IEEE Open J. Commun. Society, vol. 1, pp. 798-807, June 2020.

[5] Q. Wu and R. Zhang, "Intelligent reflecting surface enhanced wireless network via joint active and passive beamforming," IEEE Trans. Wireless Commun., vol. 18, no. 11, pp. 5394-5409, Nov. 2019.

[6] —, "Towards smart and reconfigurable environment: Intelligent reflecting surface aided wireless network," IEEE Commun. Mag., vol. 58, no. 1, pp. 106-112, Nov. 2020.

[7] Z. Abdullah, G. Chen, S. Lambotharan, and J. A. Chambers, "A hybrid relay and intelligent reflecting surface network and its ergodic performance analysis," IEEE Wireless Commun. Lett. (Early Access).

[8] Y. Liu, et al. "Reconfigurable intelligent surfaces: Principles and opportunities." arXiv:2007.03435.

[9] M. Cui, G. Zhang, and R. Zhang, "Secure wireless communication via intelligent reflecting surface," IEEE Wireless Commun. Lett., vol. 8, no. 5, pp. 1410-1414, May 2019.

[10] Z. Chu, W. Hao, P. Xiao, and J. Shi, "Intelligent reflecting surface aided multi-antenna secure transmission," IEEE Wireless Commun. Lett., vol. 9, no. 1, pp. 108-112, Sep. 2020.

[11] X. Guan, Q. Wu, and R. Zhang, "Intelligent reflecting surface assisted secrecy communication: Is artificial noise helpful or not?" IEEE Wireless Commun. Lett., vol. 9, no. 6, pp. 778-782, Jan. 2020.

[12] X. Yu, D. Xu, Y. Sun, D. W. K. Ng, and R. Schober, "Robust and secure wireless communications via intelligent reflecting surfaces," IEEE J. Sel. Areas Commun. (Early Access), 2020.

[13] H. Wang, J. Bai, and L. Dong, "Intelligent reflecting surfaces assisted secure transmission without eavesdropper's CSI," IEEE Sig. Process. Lett., vol. 27, pp. 1300-1304, Jul. 2020.

[14] L. Yang, Y. Jinxia, W. Xie, M. Hasna, T. Tsiftsis, and M. Di Renzo, "Secrecy performance analysis of RIS-aided wireless communication systems," IEEE Trans. Vehi. Technol. (Early Access), 2020.

[15] M. Badiu and J. P. Coon, "Communication through a large reflecting surface with phase errors," IEEE Wireless Commun. Lett., vol. 9, no. 2, pp. 184-188, Oct. 2020.

[16] D. Li, "Ergodic capacity of intelligent reflecting surface-assisted communication systems with phase errors," IEEE Commun. Lett., vol. 24 no. 8, pp. 1646-1650, Aug. 2020.

[17] T. Wang, G. Chen, J. P. Coon, and M. Badiu, "Study of intelligent reflective surface assisted communications with one-bit phase adjustments,' in proc. IEEE Globecom, Taiwan, China, pp. 1-6, 2020.

[18] P. Xu, G. Chen, Z. Yang, and M. Di Renzo, "Reconfigurable intelligent surfaces assisted communications with discrete phase shifts: How many quantization levels are required to achieve full diversity?" arXiv: 2008.05317

[19] W. Liu, Z. Ding, T. Ratnarajah, and J. Xue, "On ergodic secrecy capacity of random wireless networks with protected zones," IEEE Trans. Vehi. Technol., vol. 65, no. 8, pp. 6146-6158, Aug. 2016.

[20] Z. Ding, R. Schober, and H. V. Poor, "On the impact of phase shifting designs on IRS-NOMA," IEEE Wireless Commun. Lett. (Early Access).

[21] I. S. Gradshteyn and I. M. Ryzhik, Table of Integrals, Series and Products, 6th ed. New York, NY, USA: Academic, 2000.

[22] S. M. Ross, Introduction to probability models, 10th ed. Academic, Amsterdam, 2010.

[23] S. Sanayei and A. Nosratinia, "Opportunistic beamforming with limited feedback," IEEE Trans. Wireless Commun., vol. 6, no. 8, pp. 2765-2771, Aug. 2007. 